\title{
Enzymatic characterization of a trypsin-like serine protease encoded by the genome of Cell fusing agent virus
}

\author{
Christophe N. Peyrefitte - Boris A. M. Pastorino • \\ Marc Grandadam · Dominique Rolland • \\ Hugues J. Tolou · Maël Bessaud
}

Received: 29 August 2006/ Accepted: 12 October 2006/ Published online: 2 December 2006

(C) Springer Science+Business Media, LLC 2006

\begin{abstract}
Cell fusing agent virus (CFAV) is a positive strand RNA insect virus first isolated from a mosquito cell line. Based on viral morphology, phenotypic and phylogenetic studies, CFAV had been tentatively assigned to the genus Flavivirus (family Flaviviridae). The determination of the CFAV polyprotein complete sequence showed a putative serine protease domain analogue to the flaviviral NS2B/NS3 complex. This complex had been extensively studied, because it represented one of the main targets for antiflavivirus therapy development. We report herein the biochemical characterization of CFAV $\triangle \mathrm{NS} 2 \mathrm{~B}-$ NS3pro protease complex. CFAV polyprotein sequence was computationally analysed to identify the amino-acid regions involved in protease activity. We designed, expressed and purified a catalytically active protease whose enzymatic properties were determined using fluorogenic substrates. Our results showed that, despite the low level of conservation of its amino-acid sequence, CFAV protease exhibited physico-chemical properties of other flaviviruses (high $\mathrm{pH}$ value requirement for optimal activity, inhibition by salt and preference for substrates featuring a basic residue at $\mathrm{P}_{1}$ position).
\end{abstract}

C. N. Peyrefitte - B. A. M. Pastorino - M. Grandadam • H. J. Tolou - M. Bessaud (\)

Unité de virologie tropicale, Institut de médecine tropicale du Service de santé des armées, BP 46, 13998 Marseille armées, France

e-mail: publi.viro@laposte.net

D. Rolland

Laboratoire de recherche et développement, bioMérieux, chemin de l'Orme, 69280 Marcy l'Etoile, France
Keywords Cell fusing agent virus · Flavivirin · Flavivirus · NS2B · NS3 - Trypsin-like serine protease

\section{Introduction}

Cell fusing agent virus (CFAV), an insect virus, was isolated from an Aedes aegypti mosquito cell line 30 years ago [1]. CFAV-infected Aedes albopictus cell lines formed a massive syncytium while vertebrate cells could not be infected [1]. This virus was also isolated from a natural mosquito pool in Puerto Rico [2]. Virions are enveloped, spherical of approximately $50 \mathrm{~nm}$ in diameter and contain a single, positive-sense singlestranded RNA genome. These characteristics suggested that CFAV was related to the Flavivirus genus (family Flaviviridae), in spite of the absence of any serological cross-reactivity with the other flaviviruses [3]. Complete genomic sequencing confirmed this hypothesis by revealing a single open reading frame that encodes a polyprotein containing the flaviviral enzymatic domains [4].

Phylogenetic studies showed that CFAV is distantly related to the other flaviviruses [5-7] and is part of a distinct cluster inside the genus [8]. The distant relationship suggests CFAV evolved separately from the flavivirus common ancestor.

Cammisa-Parks et al. have reported the identification of a putative protease motif contained in the $\mathrm{N}$ terminal portion of CFAV NS3 protein [4]. Their sequence analysis suggested a CFAV protease cleavage specificity slightly different from the other flavivirus proteases [4]. The study of CFAV phenotypic characteristics and biochemical properties of its enzymes is of 
particular interest because of CFAV original position in the Flavivirus genus.

In previous works [9-11], we have focused on the flavivirus-encoded protease. This protease consists of two viral proteins: NS3, which contains the conserved catalytic triad of serine proteases [12, 13], and NS2B, a membrane-bound protein required as a co-factor for enzymatic activity $[14,15]$. This binary complex is involved in catalysing several cleavages inside the viral polyprotein [16-22]. The essential role of the viral protease in flavivirus replication makes the protease an attractive target for antiviral drug development [23].

We report herein the biochemical characterization of CFAV protease. The CFAV polyprotein sequence was computationally analysed to identify the aminoacid regions involved in protease activity. Based on our results, we designed, expressed and purified a catalytically active protease whose enzymatic properties were determined using fluorogenic substrates.

\section{Material and methods}

Amino-acid sequences used in this work were available on GenBank, except those of Bussuquara virus (BSQV), St.-Louis encephalitis virus (SLEV) and Zika virus (ZIKV), provided by Gilda Grard (Laboratoire de virologie moléculaire, tropicale et transfusionelle, $\mathrm{Fa}$ culté de médecine de la Timone, Marseille, France) and Yellow fever virus (YFV), determined in our laboratory.

Identification of NS2B and NS3pro encoding sequences was performed by alignment of the amino-acid sequences using Clustal_X 1.81 software [24]. Hydrophobicity profile of NS2B was generated using Vector NTI Suite v.7 (InforMax Inc., Bethesda, United States). Transmembrane helix was predicted using different web servers (Table 1).

Table 1 Web servers used to predict transmembrane helices in CFAV NS2B

\begin{tabular}{ll}
\hline Name & Weblink \\
\hline HMMTop & http://www.enzim.hu/hmmtop/index.html \\
PRED- & http://www.athina.biol.uoa.gr/PRED-TMR/ \\
TMR & http://www.sosui.proteome.bio.tuat.ac.jp/ \\
SOSUI & sosui_submit.html \\
& http://www.bioweb.pasteur.fr/seqanal/interfaces/ \\
TMAP & tmap.html \\
& http://www.cbs.dtu.dk/services/TMHMM/ \\
TMHMM & http://www.ch.embnet.org/software/ \\
TMpred & TMPRED_form.html \\
& http://www.athina.biol.uoa.gr/bioinformatics/ \\
waveTM & waveTM/input.html \\
&
\end{tabular}

The CFAV cDNA encompassing NS2B-NS3 region was provided by Xavier de Lamballerie (Laboratoire de virologie moléculaire, tropicale et transfusionelle). The vectors encoding CFAV $\triangle$ NS2B-NS3pro and $\triangle$ NS2B-NS3proHDA were obtained following the methods previously described [9] (primer sequences are available on request). Recombinant proteins were expressed and purified and enzymatic assays were carried out as previously reported [9]. Five substrates were commercially available (Orpegen Pharma, Heidelberg, Germany) and six were synthesized by JPT Peptide Technologies GmbH (Berlin, Germany). All but one substrates had a purity higher than $95 \%$. Experimental initial velocities $\left(V_{\mathrm{i}}\right)$ were determined using SoftMax Pro software (Molecular Devices, SaintGrégoire, France).

\section{Results and discussion}

In our alignment, the CFAV NS2B $\mathrm{D}^{1303}$ residue was found aligned with the N-terminal residue of NS2B protein of the other flaviviruses, whereas CFAV NS2B $\mathrm{R}^{1451}$ was aligned with $\mathrm{C}$-terminal residue. The $\mathrm{N}$-terminal limit of CFAV NS2B protein was found different from Cammisa-Parks et al. proposition ( $\mathrm{D}^{1303}$ instead of $\mathrm{H}^{1328}$ ). Based on the same alignment, CFAV NS3 protein sequence was identified from $S^{1452}$ through $R^{2038}$.

The hydrophobicity profile of CFAV NS2B protein showed a central $\sim 50$ amino-acid residue-long hydrophilic domain surrounded by hydrophobic regions (Fig. 1A). Similar hydrophobicity plots have been observed in other flaviviruses [9, 25, 26].

In silico analysis predicted three different models in which CFAV NS2B was a membrane-bound protein (Fig. 1B). The TMAP software predicted 2 membrane helix, HMMTop, TM-PRED and waveTM predicted 3 helix while PRED-TMR, SOSUI and TMHMM predicted a 4-helix model. Depending on the used software, the residues $\mathrm{Y}^{44}, \mathrm{M}^{45}, \mathrm{Q}^{47}$ or $\mathrm{V}^{50}$ were predicted to be the $\mathrm{N}$-terminal limit of the soluble domain. In contrast, the C-terminal limit was unclear, regarding the divergent results obtained (near residue 85 using PRED-TMR, SOSUI and TMHMM, near residue 100 with HMMTop, TMAP, TMPred and waveTM). These variable results can be explained by the different methods used to predict protein structure in the different software $[27,28]$. The CFAV V ${ }^{50}-S^{96}$ region was found aligned with the $\Delta \mathrm{NS} 2 \mathrm{~B}$ sequences designed in our previous works $[9,10]$ (data not shown), and was cloned as CFAV $\triangle$ NS2B in this work.

The CFAV NS3 protein sequence analysis showed the four boxes conserved amongst the serine protease 
Fig. 1 Hydrophobicity profile

(A) and schematic representation of the organisation of CFAV NS2B protein predicted using different web servers (a: HMMTop, b: PREDTMR, c: SOSUI, d: TMAP, e: TMHMM, f: TMpred, g: waveTM) (B). The solid boxes indicate the predicted transmembrane domains of NS2B. The lower scheme shows the hydrophilic domain cloned as $\triangle \mathrm{NS} 2 \mathrm{~B}$

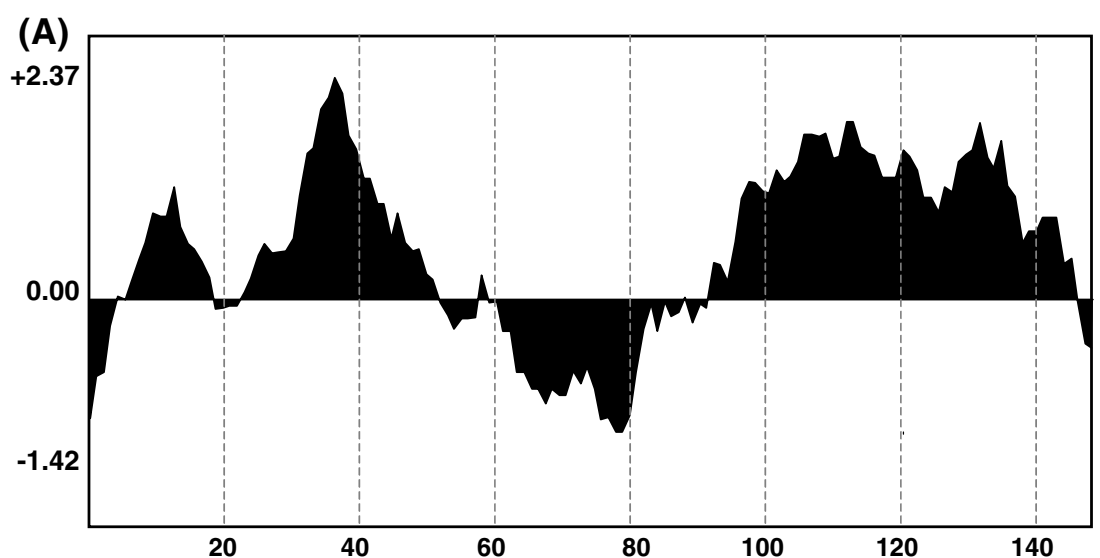

(B)

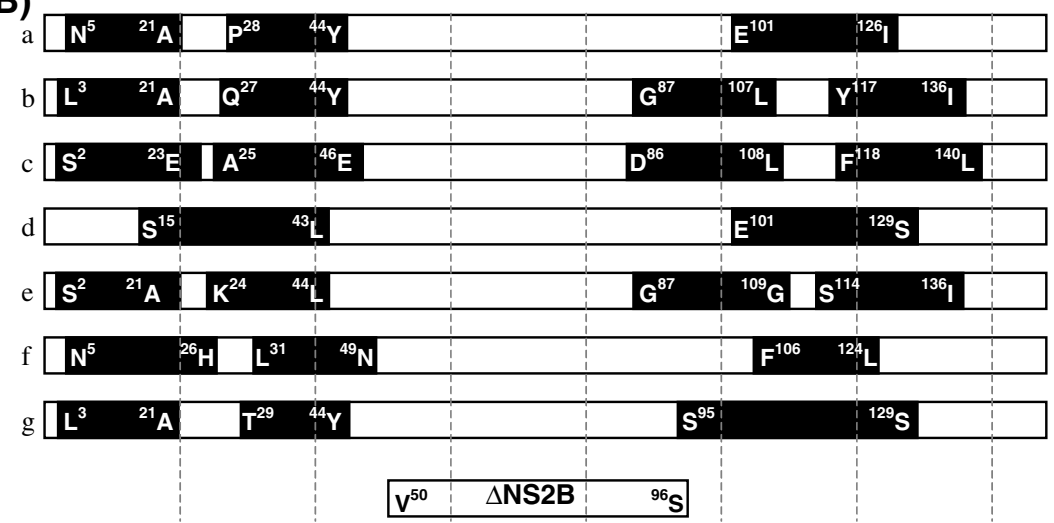

family $[10,13,29]$ and particularly the $H, D$ and $S$ residues that form the catalytic triad of serine proteases (Fig. 2).

The third box was found to contain a $\mathrm{D}^{132}$ residue located six positions upstream the catalytic triad-involved $S$ residue, which could be the $\mathrm{D}^{189}$ trypsin analogue. This pattern, observed in all NS3 flavivirus $[10,12,13,30]$ except in Tamana bat virus [31], was shown to be responsible for the trypsin-like proteases specificity towards basic substrates [32, 33]. The involvement of this $\mathrm{D}$ residue in substrate binding has been experimentally demonstrated for the WNV protease [34]. These results suggested that the CFAV protease targeted natural cleavage sites displaying a basic residue at $\mathrm{P}_{1}$ position (according to Schechter and Berger's nomenclature [35]).

The sequence alignment showed several conserved residues amongst the flavivirus, except in CFAV and in $\mathrm{KRV}$, two viruses phylogenetically close together [36]. Some of these positions (see exclamation marks in Fig. 2) are particularly outstanding such as $F^{151}$ and $\mathrm{F}^{154}$ in the fourth homology box, known to contain substrate binding residues [36]. All flavivirus proteases boxes contained a GLYGNG conserved sequence.
However, the CFAV NS3 protein displayed an unusual GFYGFG sequence, which is also observed in the KRV NS3 protein. Moreover, CFAV and KRV proteases featured a GSSGSP in the serine-protease conserved motif GxSGxP (box 3) while the other flavivirus proteases exhibited a GTSGSP [12]. Surprisingly, this GSSGSP pattern was also found in the BSQV sequence, a virus phylogenetically far away from CFAV and KRV. These changes likely imply differences in the CFAV protease enzymatic properties.

The CFAV and KRV protein sequences showed a $\mathrm{G}^{168}$ instead of the conserved $\mathrm{Q}$ residue found to be the last residue conserved in the flavivirus minimal domain [37]. The CFAV $\mathrm{K}^{170}$ residue, located two positions downstream, was the C-terminal limit for cloning, according to the previously reported strategy $[9,10]$.

The CFAV protease complex was expressed as a single protein according to the expression strategy previously reported $[9,10,38-43]$. The NS3pro sequence was fused with $\triangle \mathrm{NS} 2 \mathrm{~B}$ via the C-terminal residues of NS2B. To avoid a NS2B-NS3 the cis-cleavage of the NS2B-NS3 junction, the last NS2B residue was substituted by an A. A similar non-cleavable construct 


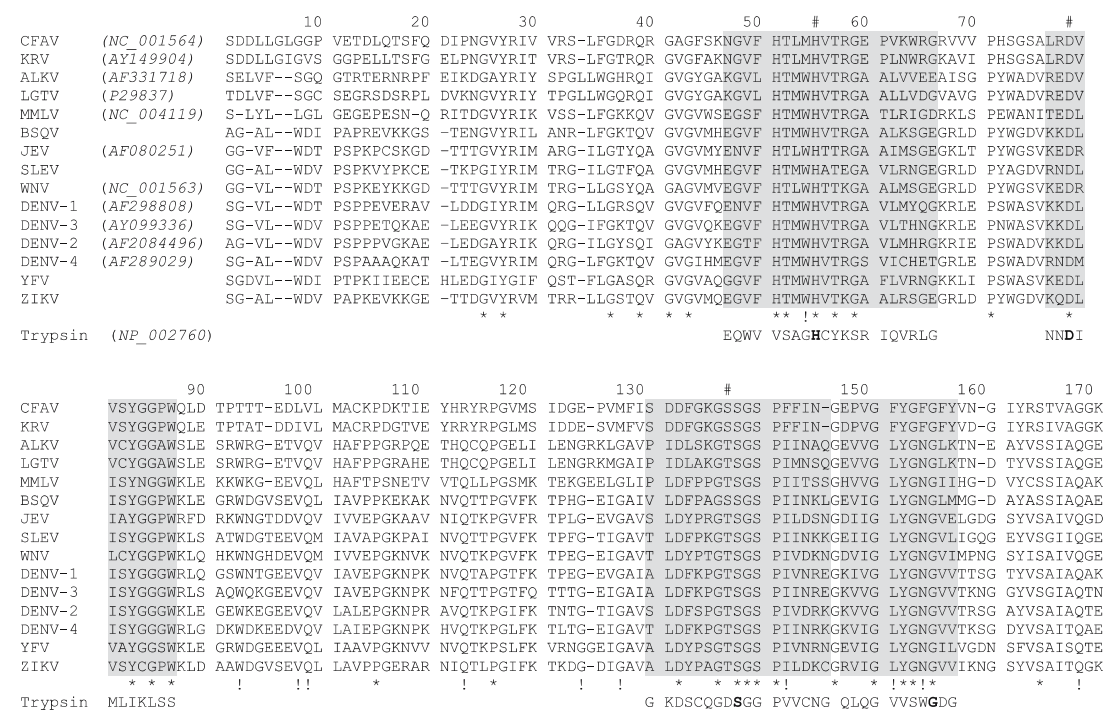

Fig. 2 Sequence alignment of NS3pro region of several flaviviruses (KRV: Kamiti River virus; ALKV: Alkhurma virus; LGTV: Langat virus; MMLV: Montana Myotis leukoencephalitis virus; JEV: Japanese encephalitis virus; WNV: West-Nile virus; DENV: Dengue virus). GenBank accession numbers are indicated in brackets. The four similarity boxes are highlighted in

( $\triangle$ NS2B-NS3proHDA) containing a $\mathrm{S} \rightarrow$ A mutation inside the catalytic triad was engineered and used as the negative control.

The CFAV recombinant proteins were found to be mainly produced as insoluble inclusion bodies (data not shown), a frequent observation for flaviviral proteases expressed under similar conditions $[9,10,40,44$, 45].

After $\mathrm{Ni}^{2+}$-affinity chromatography, Coomassie blue staining and Western blotting, the protein expression pattern was similar to those previously reported while using the same conditions [9, 10]. High molecular contaminants $(\sim 75 \mathrm{kDa})$, found both in $\triangle \mathrm{NS} 2 \mathrm{~B}-$ NS3pro and $\triangle$ NS2B-NS3proHDA (data not shown), were identified as bacterial proteins with no reported protease domain [10]. The low molecular weight bands detected using anti-V5 antibody (which target C-terminal extremity of the recombinant proteins) on $\triangle$ NS2B-NS3pro Western blotting (Fig. 3, lane A) were assumed to be $\Delta$ NS2B-NS3pro proteolysis products. Such cleavage fragments were previously described in other reports $[9,10,38,39,42]$. The absence of such contaminants accompanying the inactive $\triangle$ NS2BNS3proHDA recombinant protein (Fig. 3, lane B) implies that the truncated forms of $\triangle$ NS2B-NS3pro were made by auto-proteolysis; they were not expected to have an effect on processing the synthetic substrates [42].

The purified recombinant CFAV proteolytic activity was assayed in a fluorogenic assay using 7-amino-4- grey. The catalytic triad residues are marked with hashes (\#). Asterisks (*) indicate the residues conserved in all the aligned sequences; exclamation marks (!) indicate the residues conserved in all the aligned sequences except in CFAV and KRV ones. Residues of human trypsin (GenBank accession no. NP_002760) that belong to the similarity boxes are also displayed

methylcoumarine-labelled tripeptides. This assay had previously been used to study the activity of flavivirus proteases in vitro [10, 40, 46, 47].

No activity was detected using the catalytically inactive $\triangle \mathrm{NS} 2 \mathrm{~B}-\mathrm{NS} 3$ proHDA; this result demonstrate the absence of contaminating bacterial serine protease in the final protein preparation.

The $\mathrm{pH}$, ionic strength and temperature dependence of CFAV $\triangle$ NS2B-NS3pro activity was studied using $t$-butyloxycarbonyl-glycyl- ${ }_{L}$-lysyl ${ }_{-}{ }$-arginine 4 -methylcoumaryl-7-amide (GKR-MCA) $100 \mu \mathrm{M}$. A pH optimum was found at 10.5 (Fig. 4A): the enzyme was about 1.5-fold more active in cleaving GKR-MCA at this $\mathrm{pH}$ than at a $\mathrm{pH}$ of 10.0 or 11.0. This result was consistent with the high $\mathrm{pH}$ required for maximal in vitro $\Delta$ NS2B-NS3pro-synthetic substrates processing $[9,10,38,42,48]$. However, the optimal value observed for CFAV protease (10.5) is higher than for other flaviviral protease previously reported (8.5-9.5, depending on the virus). The CFAV protease activity was inhibited by $\mathrm{NaCl}$ (Fig. 4B): its activity dropped down approximately twofold at $\mathrm{NaCl} 250 \mathrm{mM}$ compared to $\mathrm{NaCl} 10 \mathrm{mM}$. This result, already reported for other flavivirus proteases $[9,10,38,42,44]$, can be explained by the importance of the electrostatic interactions in serine protease substrate binding. Over the examined range, temperature optimum was determined to be $28^{\circ} \mathrm{C}$ (Fig. 4C). This optimum could be related with the ability of this virus to infect mosquito cells, whose optimal temperature for in vitro culture is also $28^{\circ} \mathrm{C}$. 
A

B

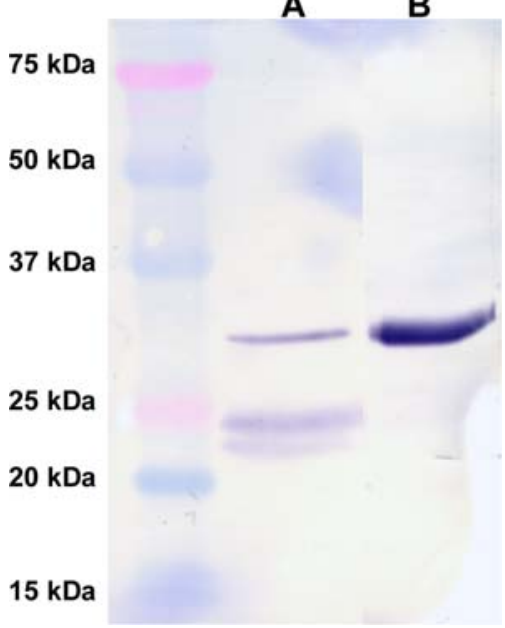

Fig. 3 Analysis by SDS-PAGE and Western blotting of $\triangle$ NS2BNS3pro (A) and $\triangle$ NS2B-NS3proHDA (B) recombinant proteins after immobilized-metal affinity chromatography

However, our previous work showed that temperature optima determined for the other flaviviral proteases did not correlate with neither viral phenotypic properties nor the conditions used to propagate the viruses [10].
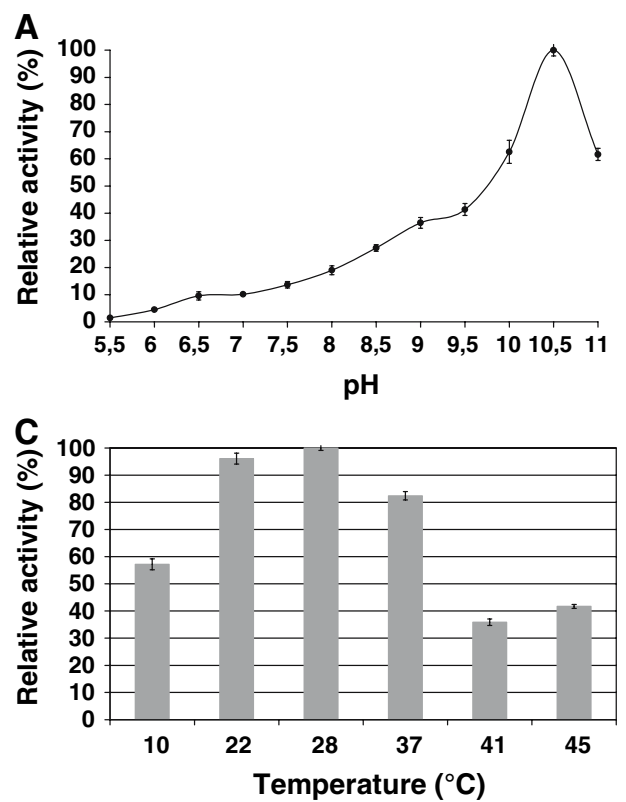

Fig. 4 Enzymatic characterization of CFAV $\triangle$ NS2B-NS3pro recombinant protein. (A) $\mathrm{pH}$ profile; for each data point, relative activity was defined as the ratio $\left(100 \times V_{\mathrm{i}}\right) /\left(V_{\mathrm{i}}\right)_{\mathrm{pH}} 10.5$. (B) Effect of ionic strength, observed in buffers containing CAPS $100 \mathrm{mM} \mathrm{pH}$ 10.5 and varying concentration of $\mathrm{NaCl}$; for each data point, relative activity was defined as the ratio $\left(100 \times V_{\mathrm{i}}\right) /\left(V_{\mathrm{i}}\right)_{10 \mathrm{mM}} \cdot(\mathbf{C})$
Among the tested substrates, the higher protease activity was observed using GKR-MCA (Fig. 4D). The use of different substrates featuring basic residues at $\mathrm{P}_{2}$ and $\mathrm{P}_{1}$ positions showed a modulation of protease activity. The protease activity was greatest when the $\mathrm{P} 2$ position was taken up by a lysine residue (compare GKR/GRR and LKR/LRR). The P3 and P1 residues were also found to influence the protease activity (compare GKR/LKR, GRR/LRR, GRR/QRR and GRR/GRK). No activity was observed using AGAMCA and AGG-MCA, two substrates that did not contain any basic residue.

Cammisa-Parks et al. predicted CFAV polypeptide cleavage sites. Some of the suggested sites, such as KA, NR or RA sequences at $\mathrm{P}_{2}-\mathrm{P}_{1}$ positions were unusual. Based on our own sequence analysis, we propose that the NS4B-NS5 cleavage occurs between R and A residues of the predicted site (FR-A, positions $\mathrm{P}_{2}-\mathrm{P}_{1}-\mathrm{P}_{1}{ }^{\prime}$ ). In an attempt to test the ability of CFAV $\triangle$ NS2BNS3pro to cleave substrates featuring FR and NR sequence at $\mathrm{P}_{2}-\mathrm{P}_{1}$ positions, the substrates GFR-MCA and GNR-MCA were synthesized. Our result using GFR-MCA provided evidence that CFAV protease was able to cleave this substrate, in spite of the lack of $\mathrm{P}_{2}$ basic residue. Unfortunately, GNR-MCA purity was too low $(<70 \%)$ to allow its use in our study.
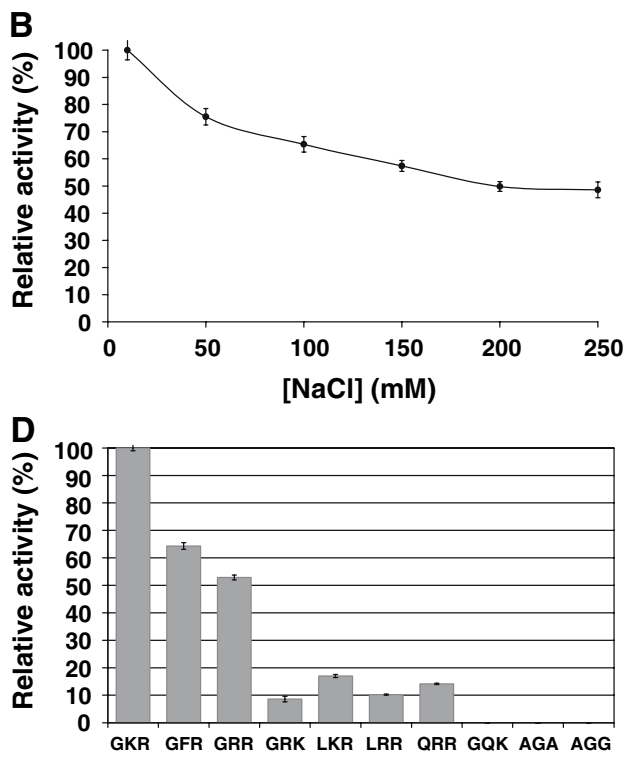

Temperature profile, performed in CAPS $100 \mathrm{mM} \mathrm{pH} 10.5, \mathrm{NaCl}$ $10 \mathrm{mM}$; for each data point, relative activity was defined as the ratio $\left(100 \times V_{\mathrm{i}}\right) /\left(V_{\mathrm{i}}\right)_{28^{\circ} \mathrm{C}}$. (D) Substrate preference assay, carried out in CAPS $100 \mathrm{mM} \mathrm{pH} 10.5, \mathrm{NaCl} 10 \mathrm{mM}$ at $28^{\circ} \mathrm{C}$. Substrate concentration was $100 \mu \mathrm{M}$; for each data point, relative activity was defined as the ratio $\left(100 \times V_{\mathrm{i}}\right) /\left(V_{\mathrm{i}}\right)_{\mathrm{GKR}}$. Error bars indicate $\pm \mathrm{SD}$ 
Taken together, these results demonstrate the requirement of a basic residue at $\mathrm{P}_{1}$ position. As discussed above, this could be explained by the role of $\mathrm{D}^{132}$ in substrate binding. The absence of protease activity towards GQK-MCA suggests that the presence of a basic residue at $P_{1}$ position is necessary but not sufficient for CFAV protease activity. The involvement of the $\mathrm{P}_{3}$ and $\mathrm{P}_{2}$ sites in substrate specificity was previously established for DENV proteases [41, 43].

In this work, we confirmed that the CFAV genome encodes a trypsin-like serine protease that shares many features with other flavivirus proteases despite a low level of amino-acid sequence conservation. Our results suggest that the CFAV $\triangle$ NS2B-NS3pro protease functions optimally in an elevated $\mathrm{pH}$ environment, is inhibited by high salt concentrations and demonstrates a preference for substrates that feature basic residues at the $\mathrm{P}_{1}$ position. These features are common to many other flavivirus proteases.

Acknowledgements The authors are indebted to Xavier de Lamballerie and Gilda Grard, who provided the CFAV cDNA and some sequences used in this study, thanked Henriette Puggelli for primer synthesis, Sylvie Lévêque for excellent technical assistance and Jon Davis for manuscript reviewing.This work was funded by the French Armed Forces Medical Service and the French Délegation générale pour l'armement (contrat d'objectif 05CO008-05).The opinions and assertions contained herein are those of the authors and are not to be construed as official or reflecting the views of the French Armed Forces Medical Service or the French Army at large.

\section{References}

1. V. Stollar, V.L. Thomas, Virology 64, 367-377 (1975)

2. S. Cook, S.N. Bennett, E.C. Holmes, R. De Chesse, G. Moureau, X. de Lamballerie, J. Gen. Virol. 87, 735-748 (2006)

3. A. Igarashi, K.A. Harrap, J. Casals, V. Stollar, Virology 74, 174-187 (1976)

4. H. Cammisa-Parks, L.A. Cisar, A. Kane, V. Stollar, Virology 189, 511-524 (1992)

5. M.S. Marin, P.M. Zanotto, T.S. Gritsun, E.A. Gould, Virology 206, 1133-1139 (1995)

6. G. Kuno, G.J. Chang, K.R. Tsuchiya, N. Karabatsos, C.B. Cropp, J. Virol. 72, 73-83 (1998)

7. F. Billoir, R. de Chesse, H. Tolou, P. de Micco, E.A. Gould, X. de Lamballerie, J. Gen. Virol. 81, 781-790 (2000)

8. S. Cook, E.C. Holmes, Arch. Virol. 151, 309-325 (2006)

9. M. Bessaud, G. Grard, C.N. Peyrefitte, B. Pastorino, D. Rolland, R.N. Charrel, X. de Lamballerie, H.J. Tolou, Virus Res. 107, 57-62 (2005)

10. M. Bessaud, B.A. Pastorino, C.N. Peyrefitte, D. Rolland, M. Grandadam, H.J. Tolou, Virus Res. 120, 79-90 (2006)

11. B.A.M. Pastorino, C.N. Peyrefitte, M. Grandadam, M.C.E. Thill, H.J. Tolou, M. Bessaud, J. Gen. Virol. 87, 3279-3283 (2006)

12. A.E. Gorbalenya, A.P. Donchenko, E.V. Koonin, V.M. Blinov, Nucl. Acids Res. 17, 3889-3897 (1989)
13. J.F. Bazan, R.J. Fletterick, Virology 171, 637-639 (1989)

14. T.J. Chambers, A. Nestorowicz, S.M. Amberg, C.M. Rice, J. Virol. 67, 6797-6807 (1993)

15. B. Falgout, M. Pethel, Y.M. Zhang, C.J. Lai, J. Virol. 65, 2467-2475 (1991)

16. C.E. Stocks, M. Lobigs, J. Virol. 72, 2141-2149 (1998)

17. B.M. Kummerer, C.M. Rice, J. Virol. 76, 4773-4784 (2002)

18. C. Lin, S.M. Amberg, T.J. Chambers, C.M. Rice, J. Virol. 67, 2327-2335 (1993)

19. T.J. Chambers, R.C. Weir, A. Grakoui, D.W. McCourt, J.F. Bazan, R.J. Fletterick, C.M. Rice, Proc. Natl. Acad. Sci. U.S.A. 87, 8898-8902 (1990)

20. F. Preugschat, C.W. Yao, J.H. Strauss, J. Virol. 64, 4364-4374 (1990)

21. G. Wengler, G. Czaya, P.M. Farber, J.H. Hegemann, J. Gen. Virol. 72, 851-858 (1991)

22. L. Zhang, P.M. Mohan, R. Padmanabhan, J. Virol. 66, 75497554 (1992)

23. P. Leyssen, E. De Clercq, J. Neyts, Clin. Microbiol. Rev. 13, 67-82 (2000)

24. J.D. Thompson, T.J. Gibson, F. Plewniak, F. Jeanmougin, D.G. Higgins, Nucl. Acids Res. 25, 4876-4882 (1997)

25. R.I. Brinkworth, D.P. Fairlie, D. Leung, P.R. Young, J. Gen. Virol. 80, 1167-1177 (1999)

26. B. Falgout, R.H. Miller, C.J. Lai, J. Virol. 67, 2034-2042 (1993)

27. C.P. Chen, A. Kernytsky, B. Rost, Protein Sci. 11, 2774-2791 (2002)

28. C.P. Chen, B. Rost, Appl. Bioinform. 1, 21-35 (2002)

29. N.D. Rawlings, A.J. Barrett, Biochem. J. 290, 205-218 (1993)

30. J.F. Bazan, R.J. Fletterick, Semin. Virol. 1, 311-322 (1990)

31. X. de Lamballerie, S. Crochu, F. Billoir, J. Neyts, P. de Micco, E.C. Holmes, E.A. Gould J. Gen. Virol. 83, 2443$2454(2002)$

32. L. Graf, C.S. Craik, A. Patthy, S. Roczniak, R.J. Fletterick, W.J. Rutter, Biochemistry 26, 2616-2623 (1987)

33. A. Rühlmann, D. Kukla, P. Schwager, K. Bartels, R. Huber, J. Mol. Biol. 77, 417-436 (1973)

34. K.J. Chappell, T.A. Nall, M.J. Stoermer, N.X. Fang, J.D. Tyndall, D.P. Fairlie, P.R. Young, J. Biol. Chem. 280, 28962903 (2005)

35. I. Schechter, A. Berger, Biochem. Biophys. Res. Commun. 27, 157-162 (1967)

36. M.B. Crabtree, R.C. Sang, V. Stollar, L.M. Dunster, B.R. Miller, Arch. Virol. 148, 1095-1118 (2003)

37. R.P. Valle, B. Falgout, J. Virol. 72, 624-632 (1998)

38. H. Li, S. Clum, S. You, K.E. Ebner, R. Padmanabhan, J. Virol. 73, 3108-3116 (1999)

39. D. Leung, K. Schroder, H. White, N.X. Fang, M.J. Stoermer, G. Abbenante, J.L. Martin, P.R. Young, D.P. Fairlie, J. Biol. Chem. 276, 45762-45771 (2001)

40. T.L. Arakaki, N.X. Fang, D.P. Fairlie, P.R. Young, J.L. Martin, Protein Exp. Purif. 25, 241-247 (2002)

41. R. Yusof, S. Clum, M. Wetzel, H.M. Murthy, R. Padmanabhan, J. Biol. Chem. 275, 9963-9969 (2000)

42. J. Li, S.P. Lim, D. Beer, V. Patel, D. Wen, C. Tumanut, D.C. Tully, J.A. Williams, J. Jiricek, J.P. Priestle, J.L. Harris, S.G. Vasudevan, J. Biol. Chem. 280, 28766-28774 (2005)

43. T.A. Nall, K.J. Chappell, M.J. Stoermer, N.X. Fang, J.D. Tyndall, P.R. Young, D.P. Fairlie, J. Biol. Chem. 279, 4853548542 (2004)

44. P. Niyomrattanakit, S. Yahorava, I. Mutule, F. Mutulis, R. Petrovska, P. Prusis, G. Katzenmeier, J.E. Wikberg, Biochem. J. 397, 203-211 (2006)

45. R. Khumthong, C. Angsuthanasombat, S. Panyim, G. Katzenmeier, J. Biochem. Mol. Biol. 35, 206-212 (2002) 
46. N. Scaramozzino, J.M. Crance, C. Drouet, J.P. Roebuck, E. Drouet, A. Jouan, D. Garin, Biochem. Biophys. Res. Commun. 294, 16-22 (2002)

47. S. Chanprapaph, P. Saparpakorn, C. Sangma, P. Niyomrattanakit, S. Hannongbua, C. Angsuthanasombat, G. Katzenmeier, Biochem. Biophys. Res. Commun. 330, 1237-1246 (2005)
48. S.A. Shiryaev, B.I. Ratnikov, A.V. Chekanov, S. Sikora, D.V. Rozanov, A. Godzik, J. Wang, J.W. Smith, Z. Huang, I. Lindberg, M.A. Samuel, M.S. Diamond, A.Y. Strongin, Biochem. J. 393, 503-511 (2006)

49. R. Khumthong, P. Niyomrattanakit, S. Chanprapaph, C. Angsuthanasombat, S. Panyim, G. Katzenmeier, Protein Pept. Lett. 10, 19-26 (2003) 